\title{
PRECISE GLOBAL DEM GENERATION BY ALOS PRISM
}

\author{
T. Tadono ${ }^{\text {a, } *, \text { H. Ishida }}{ }^{\text {a }}$, F. Oda ${ }^{\text {a }}$, S. Naito ${ }^{\text {a }}$, K. Minakawa ${ }^{\text {a }}$, H. Iwamoto ${ }^{\text {a }}$ \\ a Japan Aerospace Exploration Agency (JAXA), 2-1-1, Tsukuba, Ibaraki, 305-8505 Japan \\ - (tadono.takeo, ishida.haruyuki, oda.fumiko, naito.shino, minakawa.kenta, iwamoto.hiroyuki)@jaxa.jp
}

Commission IV, WG IV/3

KEY WORDS: Global, High resolution, DEM/DTM, Satellite, Mapping

\begin{abstract}
:
The Japan Aerospace Exploration Agency (JAXA) generated the global digital elevation/surface model (DEM/DSM) and orthorectified image (ORI) using the archived data of the Panchromatic Remote-sensing Instrument for Stereo Mapping (PRISM) onboard the Advanced Land Observing Satellite (ALOS, nicknamed "Daichi"), which was operated from 2006 to 2011.

PRISM consisted of three panchromatic radiometers that acquired along-track stereo images. It had a spatial resolution of $2.5 \mathrm{~m}$ in the nadir-looking radiometer and achieved global coverage, making it a suitable potential candidate for precise global DSM and ORI generation. In the past 10 years or so, JAXA has conducted the calibration of the system corrected standard products of PRISM in order to improve absolute accuracies as well as to validate the high-level products such as DSM and ORI.

In this paper, we introduce an overview of the global DEM/DSM dataset generation project, including a summary of ALOS and PRISM, in addition to the global data archive status. It is also necessary to consider data processing strategies, since the processing capabilities of the level 1 standard product and the high-level products must be developed in terms of both hardware and software to achieve the project aims. The automatic DSM/ORI processing software and its test processing results are also described.
\end{abstract}

\section{INTRODUCTION}

Topographical information is fundamental to any geo-spatial information and applications on Earth. Remote sensing satellites have the advantage in collecting such information because they are capable of repeated global observation.

Recently, various satellite data and their derivative datasets were provided to examine global terrains with medium and fine resolutions, by the Shuttle Radar Topography Mission (SRTM), which was spearheaded by the National Aeronautics and Space Administration (NASA) and the National GeospatialIntelligence Agency (NGA) of the United States (Farr et al., 2007). These data have 3 arcsec (approx. $90 \mathrm{~m}$ ) spacing, which only covers latitude regions within +/-60 degrees, and a "void area" still remains due to Radar shadowing and low correlations. The global digital elevation model dataset, which uses data acquired by the Advanced Spaceborne Thermal Emission and Reflection Radiometer (ASTER GDEM), was released in 2009 by NASA and the Ministry of Economy, Trade and Industry (METI), Japan, and a second version was released in 2011 (Tachikawa et al., 2011). This dataset has 1-arcsec spacing as its specification, but problems with its quality still remain. SRTM version 3 was produced by combining SRTM and GDEM, and was released in 2012.

A new global digital elevation/surface model (DEM/DSM) dataset using the data acquired by the Panchromatic Remotesensing Instrument for Stereo Mapping (PRISM) onboard the Advanced Land Observing Satellite (ALOS, nicknamed "Daichi") was launched by the Japan Aerospace Exploration Agency (JAXA) in collaboration with commercial partners NTT DATA Corp. and Remote Sensing Technology Centre of Japan (RESTEC). This project is named "ALOS World 3D", and the dataset created consists of fine resolution DSM $(0.15$ arcsec, approx. $5 \mathrm{~m}$ ) and ortho rectified image (ORI) of PRISM in global terrestrial area. It uses approx. 3 million scenes of PRISM, therefore the data processing strategies have to be considered in terms of both hardware and software to achieve the project aims. This paper describes an overview of the project as well as ALOS PRISM, the archived data status, the processing strategies including automatic processing software development, and test processing results of the feasibility study.

\section{ALOS AND PRISM}

ALOS was launched on Jan. 24, 2006, and operated very well throughout the mission target life of five years (Shimada et al., 2010). However, its operation moved to the Low Load Mode on Apr. 22, 2011 due to a power generation anomaly. This was subsequently followed by an official termination of the mission on May 12, 2011. Notwithstanding, approximately 6.5 million scenes of archived data covering the entire globe are available to users.

PRISM was an optical instrument onboard ALOS that consisted of three radiometers for nadir- (NDR), forward- (FWD), and backward- (BWD) looking, and had a $2.5 \mathrm{~m}$ spatial resolution with a $35 \mathrm{~km}$ swath width in triplet observing mode (OB1). It also had the capability to obtain NDR and BWD images in stereo observing mode (OB2) (Tadono et al., 2009). Figure 1 illustrates the PRISM observation geometries, and Table 1 summarizes the primary PRISM characteristics. Their images perform along-track stereo observation to generate precise DEM/DSM. Figure 2 shows a map of PRISM stereo scenes in the archives with less than $30 \%$ cloud cover in a scene that is approx. 3 million scenes. The dataset is basically produced using these scenes; therefore, a processing system with a huge capacity for both level 1 and DSM/ORI had to be used.

\footnotetext{
* Corresponding author. This is useful to know for communication with the appropriate person in cases with more than one author.
} 

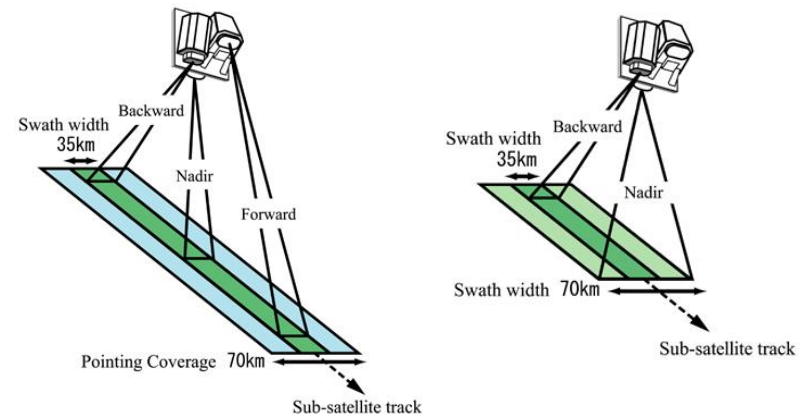

Figure 1. Observation geometries of PRISM triplet observing mode (OB1, left), and stereo (by nadir plus backward) observing mode (OB2, right).

Table 1. PRISM characteristics.

\begin{tabular}{lc}
\hline \hline \multicolumn{1}{c}{ Item } & Description \\
\hline Number of bands & 1 (Panchromatic) \\
Wavelength & $0.52-0.77$ micrometers \\
Number of optics & 3 (NDR, FWD, and BWD) \\
Base to height ratio & 1.0 (between FWD and BWD) \\
Spatial resolution & $2.5 \mathrm{~m}$ (NDR) \\
Swath width & $35 \mathrm{~km}(\mathrm{OB} 1) / 70 \mathrm{~km}(\mathrm{OB} 2)$ \\
Signal to noise ratio & $>70$ \\
MTF & $>0.2$ \\
Pointing angle & $-1.2 /+1.2$ degrees (OB1, NDR) \\
Bit length & 8 bits/pixel \\
Data rate & $960 \mathrm{Mbps}(\mathrm{OB} 1$, OB2) \\
Data compression & Lossy, JPEG extension (onboard) \\
Data downlink rate & $240 \mathrm{Mbps}(1 / 4.5$ compression) \\
\hline \hline
\end{tabular}

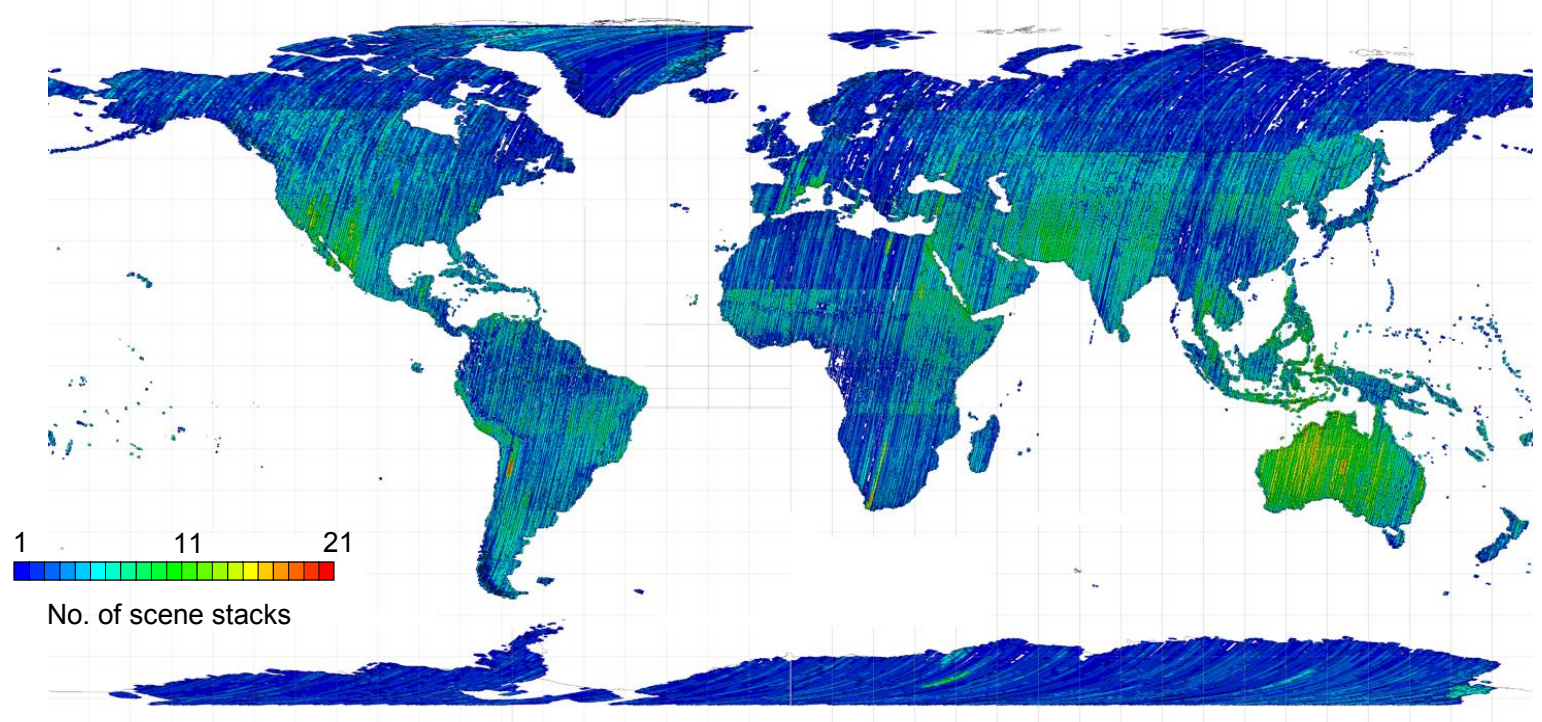

Figure 2. The status of PRISM stereo scenes in the archive with $<30 \%$ cloud cover.

Table 2. Specifications of the "ALOS World $3 D$ " dataset.

\begin{tabular}{|c|c|}
\hline Product Name & Specifications / Descriptions \\
\hline 1) Precise DSM Dataset & 1 deg. tile of lat/long frame unit \\
\hline DSM file (DSM) & $\begin{array}{l}\text { The geoid height of GRS80 ellipsoid model with ITRF97 coordination ( } m \text { unit). } \\
\text { Equivalent lat/long, Signed } 16 \mathrm{bit} \text { raster data, GeoTIFF format } \\
\text { Horizontal spacing: } 0.15 \text { arcsec (approx. } 5 \mathrm{~m} \text { ) } \\
\text { Accuracies: } 5 \mathrm{~m} \text { in height (RMSE), } 5 \mathrm{~m} \text { in geolocation (RMSE) }\end{array}$ \\
\hline Mask file (MSK) & $\begin{array}{l}\text { Masked out due to clouds, land water, and ocean in individual tile. } \\
\text { 8bit raster data, GeoTIFF format }\end{array}$ \\
\hline Stack number file (STK) & $\begin{array}{l}\text { Stacking number of the scene-frame DSM for individual pixel. } \\
\text { 8bit raster data, GeoTIFF format }\end{array}$ \\
\hline Header information (HDR) & Processing information for generating the dataset in ASCll text \\
\hline Scene list (LST) & Scene information of source scene-frame DSM in ASCII text \\
\hline $\begin{array}{l}\text { Quality assessment } \\
\text { information (QAI) }\end{array}$ & $\begin{array}{l}\text { Summary of the quality assessment information (e.g. stats of differences with } \\
\text { existing DEMs etc.) in ASCII text }\end{array}$ \\
\hline 2) ORI Dataset & Nadir image, Scene-frame unit \\
\hline Ortho rectified image (ORI) & $\begin{array}{l}\text { ITRF97 and GRS80 coordination, GeoTIFF format. } \\
\text { Horizontal spacing: } 0.075 \text { arcsec (approx. } 2.5 \mathrm{~m} \text { ) } \\
\text { Geolocation accuracy: } 5 \mathrm{~m} \text { (RMSE) }\end{array}$ \\
\hline Header file (OHR) & Source data information in ASCll text \\
\hline $\begin{array}{l}\text { 3) Correlation Coefficient Image } \\
\text { (CCI) }\end{array}$ & 8bit raster data, GeoTIFF format (Intermediate product) \\
\hline
\end{tabular}

\section{PROCESSING STRATEGY}

\subsection{Specifications of the dataset}

Table 2 summarizes the specifications of the PRISM global DSM dataset being processed. It consists of the precise DSM in 1 degree tiles of lat/long frame units, and ORI of NDR in scene bases. As mentioned before, the precise DSM has 0.15 arcsec (approx. $5 \mathrm{~m}$ ) horizontal spacing with $5 \mathrm{~m}$ height accuracy in root mean square error (RMSE) as the target accuracy. This may be achieved by stacking and mosaicking processing using multitemporal acquired scenes of DSM, as summarized in Fig. 2. The ORI is processed for NDR only due to necessity and limitation of data storage. It has 0.075 arcsec horizontal spacing, which corresponds to approx. $2.5 \mathrm{~m}$ original resolution. The correlation coefficient image $(\mathrm{CCI})$ is an intermediate product of the image matching processing in DSM generation. A summary of $\mathrm{CCI}$ is given in the quality assessment information (QAI). 


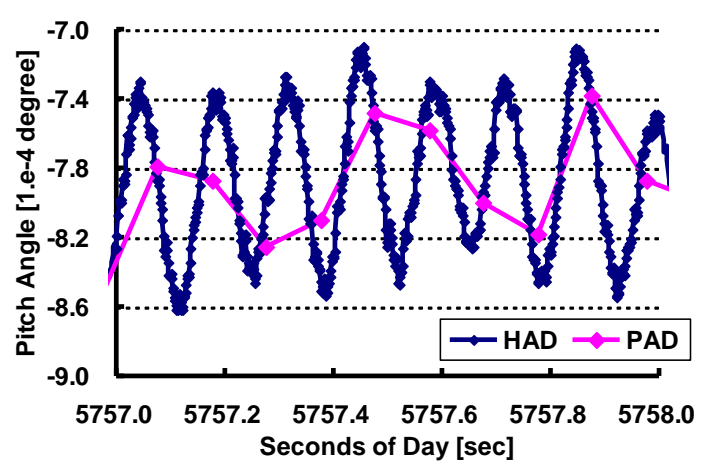

Figure 3. An example of attitude data comparison between PAD and $\mathrm{HAD}$

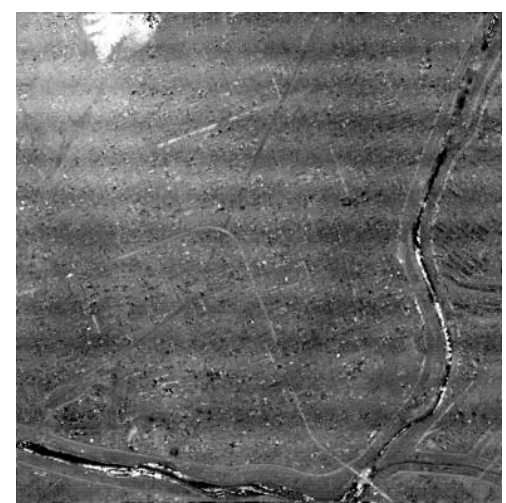

(a) DSM processing with PAD.

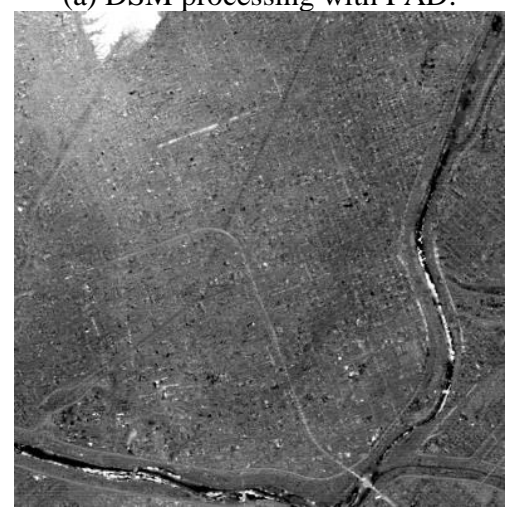

(b) DSM processing with HAD.

Figure 4. Comparison between the PRISM DSM processed with (a) PAD and (b) HAD (Takaku and Tadono, 2010).

\subsection{Level 1B1 processing}

The input data for processing PRISM DSM/ORI by our software is level $1 \mathrm{~B} 1$ of the standard processing product. It is generally processed at the Earth Observation Center (EOC) i.e., JAXA's data processing center; however, it does not have processing capacity for a huge amount of scenes.

Additionally, a problem reported by a previous study (Takaku and Tadono, 2010) is the attitude fluctuations of the satellite that sometimes cause jitter noises to appear in the generated PRISM DSM. Operationally, the attitude data of PRISM is provided at $10 \mathrm{~Hz}$ of sampling rate called the precision attitude data (PAD). It is composited the data of the star tracker (STT) and the inertial reference unit (IRU) (Iwata et al., 2008). Meanwhile, the high-frequency attitude data (HAD) is produced at a sampling rate of $675 \mathrm{~Hz}$ by compositing PAD and the angler displacement sensor (ADS) for the on-orbit technical validation purposes. Figure 3 shows an example of attitude data

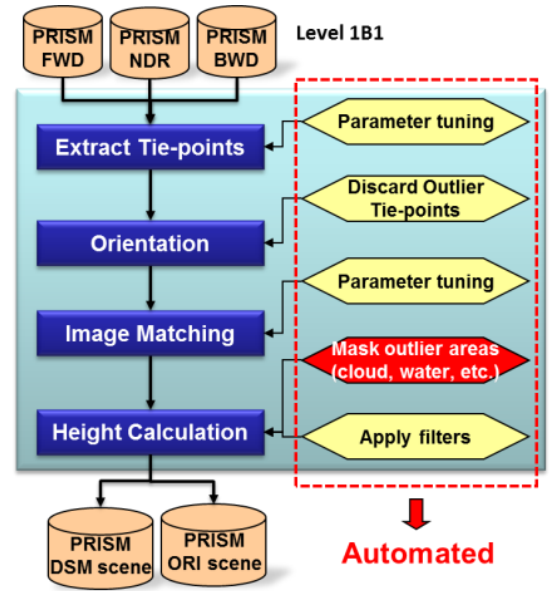

Figure 5. The processing flowchart of "DOGS-AP" software.

comparison between PAD and HAD. It can be clearly seen PAD follows HAD even though the sampling rate is different. Figure 4 shows an image comparison example of DSM processed using PAD and HAD. Jitter noises can be seen in along-track direction in the DSM processed with PAD, however such noises do not appear in the DSM processed with HAD. HAD cannot completely remove all jitter noises in DSM, but it does greatly reduce them as shown in Fig. 4. Therefore, the level 1B1 with HAD processing system was developed in the JAXA Earth Observation Research Center (EORC) to process input data for the global PRISM DSM/ORI dataset. It is estimated that this system has the processing capacity of two hundred thousand scenes per month.

\subsection{DSM/ORI dataset processing}

We are developing software called the "DSM and Ortho-image Generation Software for ALOS PRISM (DOGS-AP)" to process the scene- and manual-bases DSM and ORI using PRISM stereo image pairs (Takaku and Tadono, 2009a). DOGS-AP also has an instrument calibration function. Since the launch of ALOS, we have carried out the calibration of PRISM to improve the absolute accuracy of the standard products (Tadono et al., 2009, 2010). This result is also reflected in the DOGS-AP software. As a result, GCPs are not essential in processing PRISM DSM and ORI because its standard product has good planimetric accuracy of $6.1 \mathrm{~m}$ (RMSE) for nadir radiometer.

The generated PRISM DSMs were also validated at several test sites with different surface conditions (Takaku and Tadono, 2009b, 2010). The confirmed height accuracies of PRISM DSMs fell in the range 2.94 to $7.15 \mathrm{~m}$ (RMSE), $\sim 3.44 \mathrm{~m}$ (bias) and $\sim 6.44 \mathrm{~m}$ (standard deviation, STDEV). In addition, in a previous study we also investigated the generation of a DSM mosaic to cover large-scale areas and concluded that the bias error correction only has to consider the height, even if the GCPs were not used in processing them (Takaku and Tadono, 2011). So far, more than 6,000 scenes of PRISM DSM and ORI have been processed at JAXA for calibration, validation, and scientific research purposes. This number is small compared with 3 million of the global scale. The automatic processing algorithm had to consider expanding the area globally, which is considered based on the experiences of previous studies.

Figure 5 shows a flowchart of the DOGS-AP, and issues for realizing automatic processing. The core process functions such as orientation, image matching etc. are kept with some improvements to the original processes, whilst the most challenging process is applying the masks in clouds, snow and ice covered land areas, and water bodies. 


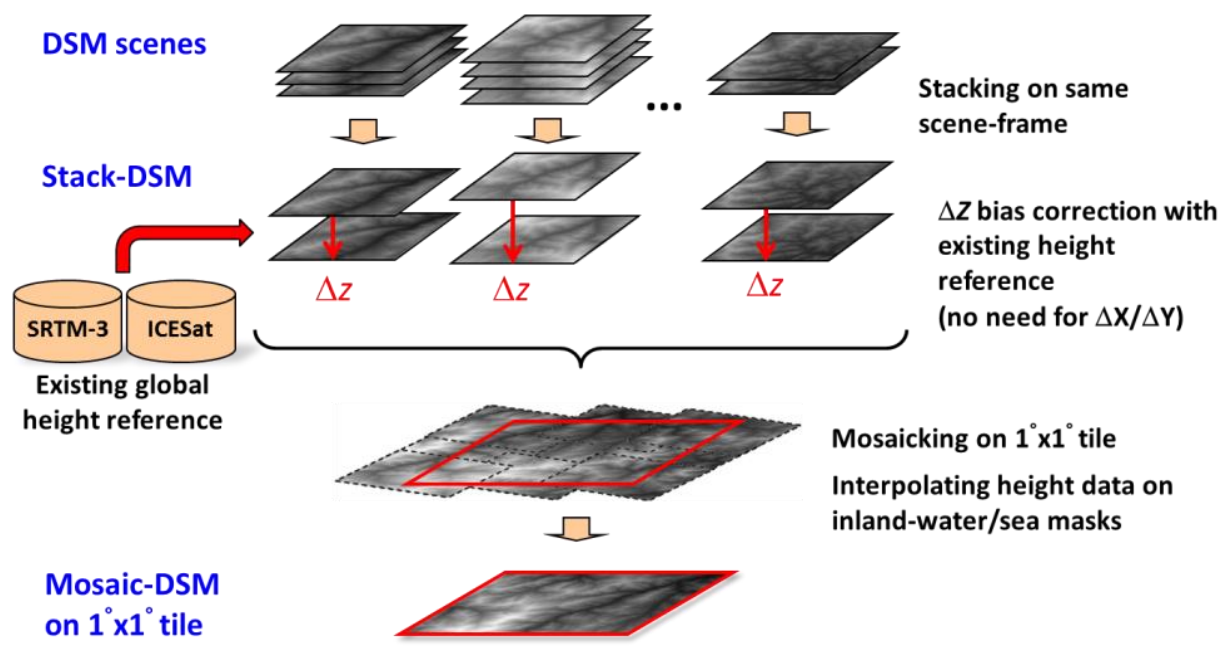

Figure 6. The concept of stacking and mosaicking processing using multi-temporal scene-based DSMs.

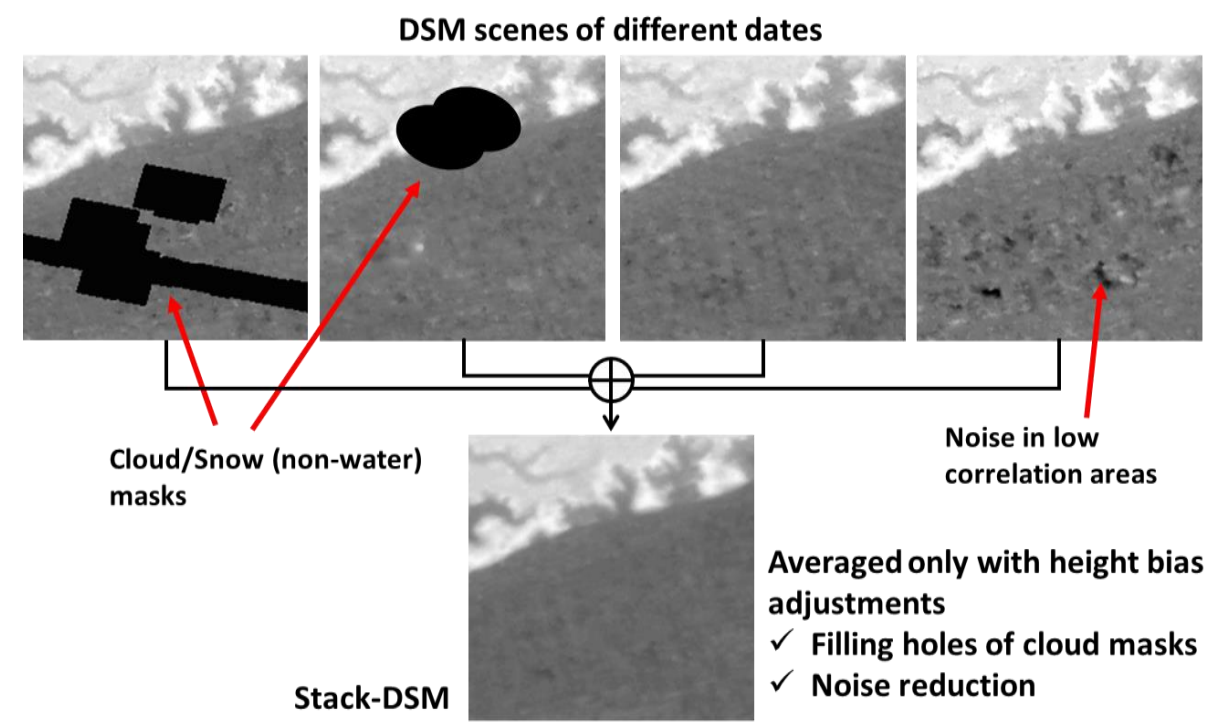

Figure 7. An example of the visual comparison between the stacked DSM and individual scene-based DSMs.

A parallel distributed processing system was developed for processing a huge number of DSM/ORI scenes. The stacking and mosaicking processing are applied after complete creation of scene-based DSMs, which are also automatically processed. Figure 6 describes the concept of DSM stacking and mosaicking processing from the scene-based DSMs. The height bias correction is only considered when applying the stacking process once the individual DSM has basically satisfied planimetric accuracy, which is evaluated using height reference data, i.e., the Ice, Cloud, and land Elevation Satellite (ICESat) data, SRTM version 2 data, etc. The height value in the stacked DSM is calculated as the averaged value of all available scenebased DSMs. Figure 7 shows an example of visual image comparison between the stacked DSM and individual scenebased DSMs, which was used in the stacking processing. The stacked DSM appears to have no holes arising from masks and low noises or even remaining masked areas or height noises in the individual DSM, so it is effective in creating a globally homogeneous DSM dataset. The stacking number depends on the area, as shown in Fig. 2. However, we are considering using available PRISM archived data as much as possible. Even so, the masks may remain in version 1 of the dataset.

The "ALOS World 3D" dataset will be released from March 2014, and is expected to complete the global dataset generation by March 2016.

\section{TEST PROCESSING}

We tested the automatic DSM/ORI processing for wide-scale areas with a large number of level 1B1 scenes to confirm the procedure, relative accuracies, processing time estimations, etc. as a feasibility study. Four test sites with varying terrain features were selected for the study as follows;

(a) Cambodian Basin: tropical forest, clouds, flat topography

(b) Senegal East: deserts, clouds, cost line

(c) Nepal East: steep mountains, clouds, snow and glaciers

(d) Sri Lanka North: the tropics, clouds, coast line

Each test site covered an area larger than $2 \times 2 \mathrm{deg}$. lat/long tiles with around 1,000 scenes of level 1B1. 22 tiles have been processed in total. It is not easy to generate precise DEM/DSM for the selected terrain features from both optical stereo and Radar interferometry from space.

Figure 8 shows the results of the test processing for each site. The left images show the locations, the middle images are processed DSM mosaics, and those on the right are height difference images of PRISM DSM minus SRTM-3 version 2, which are used as relative validations because the acquired year between SRTM and PRISM is different. Table 3 summarizes the height difference stats of PRISM DSM minus SRTM-3 for 


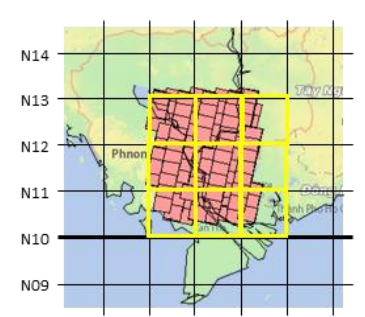

E103 E104 E105 E106 E107 E108

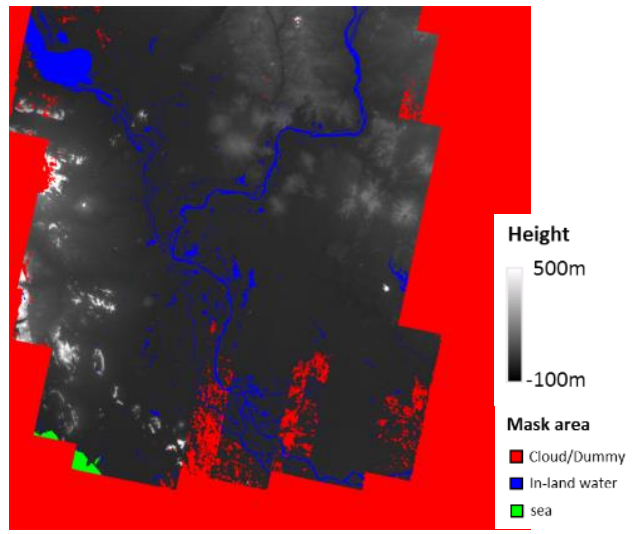

(a) Cambodian Basin
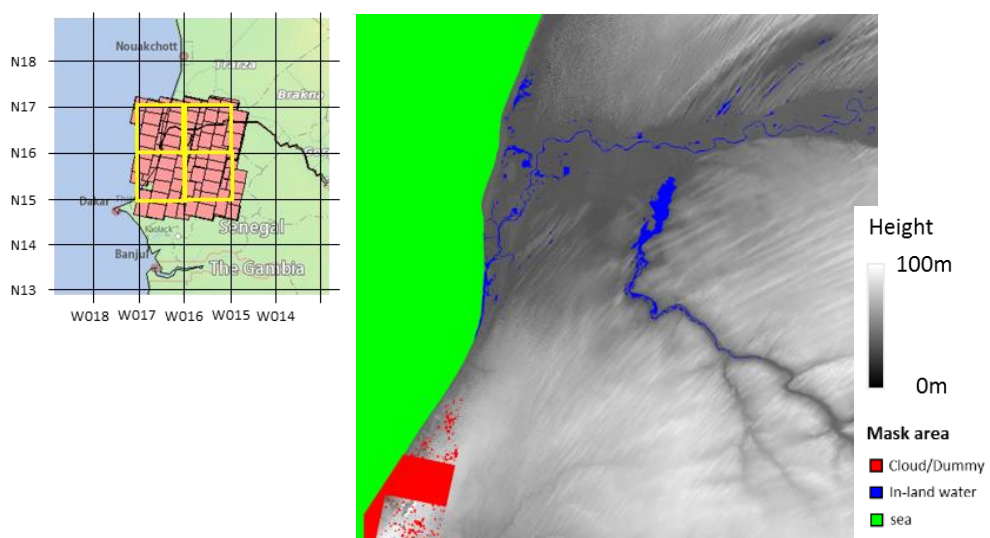

(b) Senegal North.
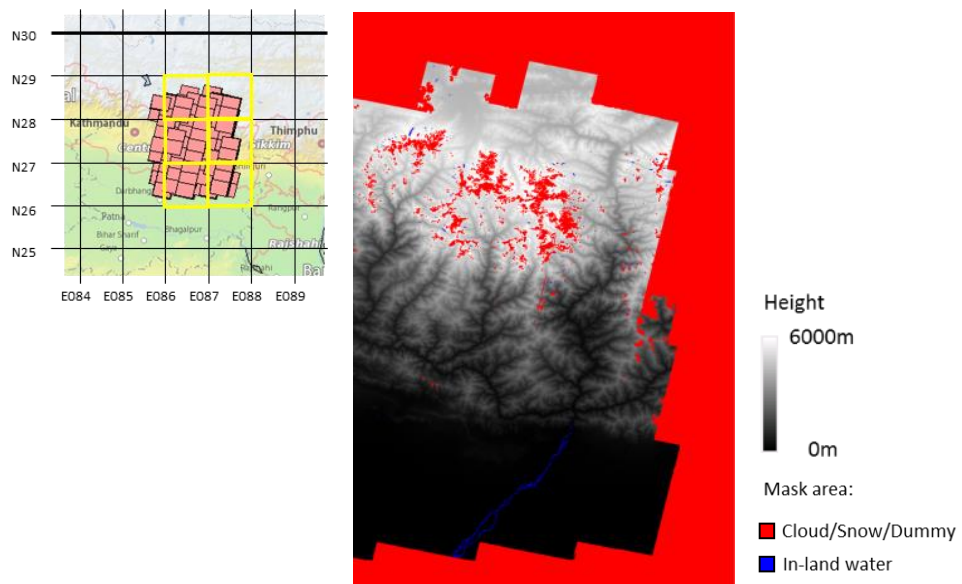

(c) Nepal East.
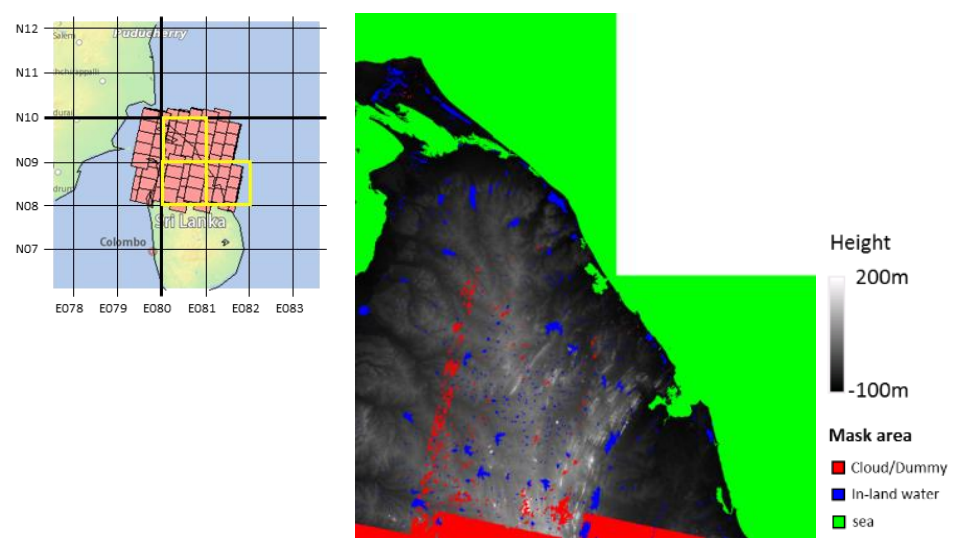

(d) Sri Lanka North.
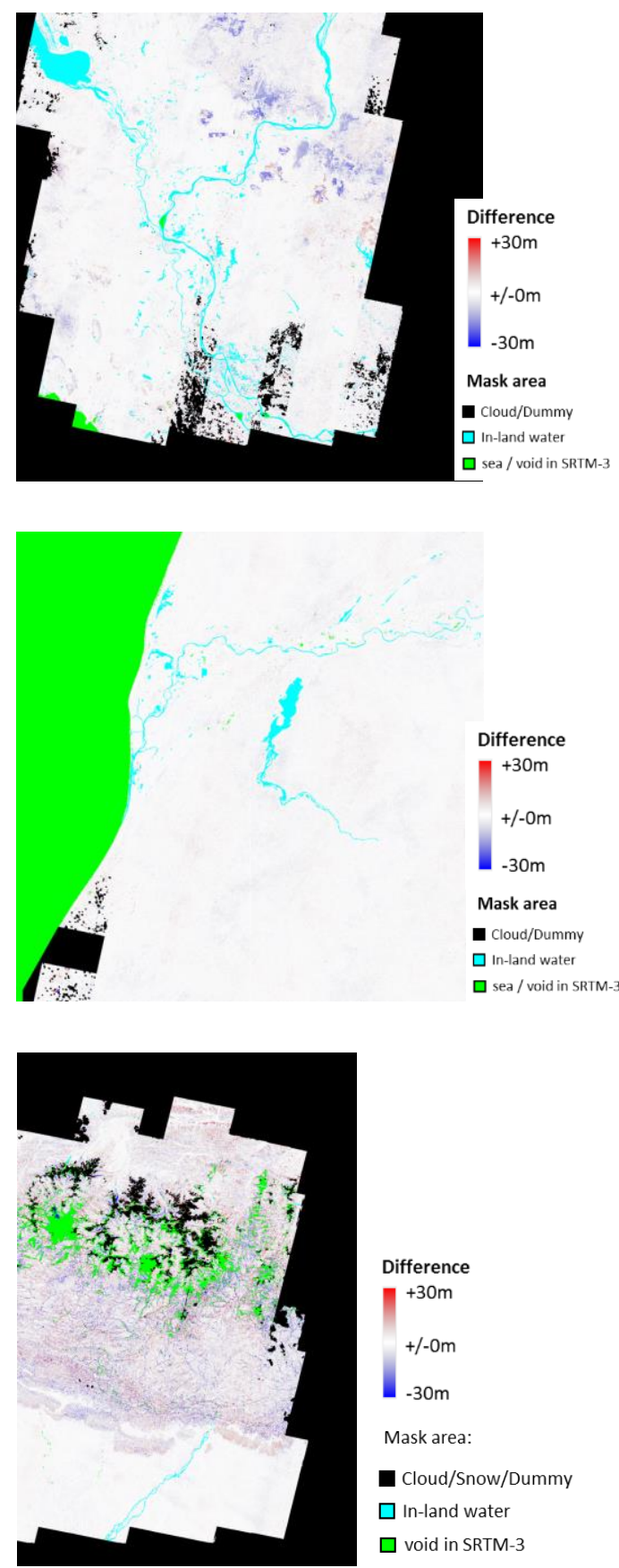

Difference

$+30 \mathrm{~m}$

$+/-0 m$

$-30 \mathrm{~m}$

Mask area:

Cloud/Snow/Dummy

$\square$ In-land water

$\square$ void in SRTM-3

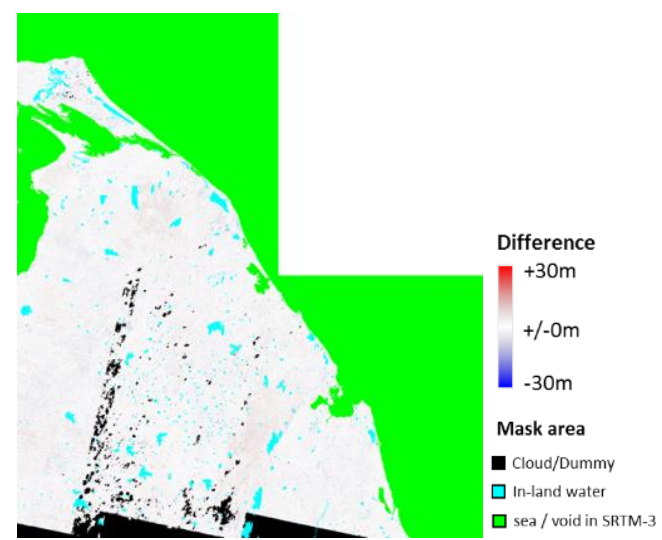

Figure 8. Test site locations (left) and the object DSM tiles (yellow squares) for the test processing, results of the large-scale area PRISM DSM (middle), and the height difference image of PRISM DSM minus SRTM-3 version 2 (right). 
Table 3. Height difference stats for each tile in test processing. (a) Cambodian Basin.

\begin{tabular}{l|r|r|r|r|r|r|}
\hline Tile & No of samples & Ave $[\mathrm{m}]$ & STDEV [m] & RMSE [m] & Max [m] & Min [m] \\
\hline N010E104 & 73586009 & -0.17 & 3.59 & 3.6 & 626 & -156 \\
N010E105 & 82687505 & 0.16 & 8.32 & 8.32 & 620 & -208 \\
N010E106 & 34874453 & -0.08 & 5.89 & 5.9 & 600 & -301 \\
N011E104 & 125560287 & -0.16 & 3.32 & 3.32 & 786 & -261 \\
N011E105 & 137181894 & -0.16 & 3.31 & 3.31 & 702 & -266 \\
N011E106 & 48968783 & -0.51 & 5.07 & 5.1 & 561 & -182 \\
N012E104 & 113446476 & -0.08 & 3.36 & 3.36 & 885 & -205 \\
N012E105 & 140181211 & -1.52 & 4.95 & 5.18 & 524 & -188 \\
N012E106 & 57735934 & -1.1 & 5.21 & 5.33 & 254 & -331 \\
\hline
\end{tabular}

(b) Senegal North.

\begin{tabular}{l|r|r|r|r|r|r|}
\hline Tile & No of samples & Ave [m] & STDEV [m] & RMSE [m] & Max [m] & Min [m] \\
\hline N015W016 & 143313857 & -0.01 & 1.91 & 1.91 & 117 & -74 \\
N015W017 & 94685510 & 0.17 & 2.46 & 2.46 & 374 & -159 \\
N016W016 & 140495007 & -0.1 & 1.97 & 1.98 & 488 & -179 \\
N016W017 & 61705303 & 0.14 & 2.25 & 2.25 & 635 & -213 \\
\hline
\end{tabular}

(c) Nepal East.

\begin{tabular}{l|r|r|r|r|r|r|}
\hline Tile & No of samples & Ave [m] & STDEV [m] & RMSE [m] & Max [m] & Min [m] \\
\hline N026E086 & 116675162 & -0.19 & 5.19 & 5.19 & 282 & -176 \\
N026E087 & 73838430 & -0.11 & 6.34 & 6.34 & 301 & -120 \\
N027E086 & 125638694 & -0.2 & 12.12 & 12.12 & 801 & -408 \\
N027E087 & 77650003 & -0.39 & 12.39 & 12.4 & 697 & -380 \\
N028E086 & 72716351 & -0.51 & 14.92 & 14.93 & 426 & -572 \\
N028E087 & 50430075 & 0.72 & 9.27 & 9.3 & 346 & -219 \\
\hline
\end{tabular}

(d) Sri Lanka North.

\begin{tabular}{l|r|r|r|r|r|r|}
\hline Tile & No of samples & Ave $[\mathrm{m}]$ & STDEV [m] & RMSE [m] & Max [m] & Min [m] \\
\hline N008E080 & 128187825 & 0.42 & 3.58 & 3.61 & 586 & -243 \\
N008E081 & 35855387 & -0.16 & 2.9 & 2.9 & 244 & -124 \\
N009E080 & 58585505 & 0.09 & 2.99 & 2.99 & 221 & -183 \\
\hline
\end{tabular}

the individual tile that shown as yellow squares in Fig. 8 . Although the stats are used for reference, the bias error is almost zero for all tiles, and 13 tiles have been confirmed to have height accuracies (RMSE) better than $5 \mathrm{~m}$ from Table 3 . We also confirmed that the processing time for 4,000 scenes by 10 nodes x 16 core CPUs was about 7 hours, which was almost the same as our expectations.

\section{CONCLUSIONS}

This paper introduced overviews of the "ALOS World 3D" dataset generation by ALOS PRISM, processing strategies including automatic processing software development, and test processing results. This project is being conducted through joint initiatives between JAXA and the private sector, utilizing results of JAXA's research and development.

The dataset consists of high resolution (5 m spacing) DSM and ORI of PRISM NDR images over a global land area, which will be able to contribute any applications related to geo-spatial information even though the data used here were acquired from 2006 to 2011. It will be distributed to commercial bases by the collaborator from March 2014. We also plan to generate a low resolution DSM dataset (30 $\mathrm{m}$ spacing, TBD) that will have the same height accuracy of $5 \mathrm{~m}$ (RMSE), and will be provided free of charge to contribute to research and science communities including the Group on Earth Observation System of Systems (GEOSS).

\section{References}

Farr, T. G., P. A. Rosen, E. Caro, R. Crippen, R. Duren, S. Hensley, M. Kobrick, M. Paller, E. Rodriguez, L. Roth, D. Seal, S. Shaffer, J. Shimada, J. Umland, M. Werner, M. Oskin, D. Burbank, and D. Alsdorf, 2007. The Shuttle Radar Topography Mission. Report on the Jet Propulsion Laboratory, California Institute of Technology, California, US

http://www2.jpl.nasa.gov/srtm/SRTM_paper.pdf (14 Jan. 2014).

Iwata, T., T. Tadono, T. Kawahara, and M. Abe, 2008. Precision Pixel Geolocation Determination for the Advanced Land Observing Satellite (ALOS). Proc. 59th International Astronautical Congress.

Shimada, M., T. Tadono, and A. Rosenqvist, 2010. Advanced Land Observing Satellite (ALOS) and Monitoring Global Environmental Change. Proc. the IEEE, 98(5), pp. 780-799.

Tachikawa, T., M. Hato, M. Kaku, and A. Iwasaki, 2011. The characteristics of ASTER GDEM version 2. Proc. IGARSS 2011, IEEE, CD-ROM.

Tadono, T., M. Shimada, H. Murakami, and J. Takaku, 2009. Calibration of PRISM and AVNIR-2 onboard ALOS "Daichi". Transactions on Geoscience and Remote Sensing, IEEE, 47(12), pp. 4042-4050.

Tadono, T, T. Iwata, M. Shimada, J. Takaku, and S. Kawamoto, 2010. Updated results of calibration and validation of PRISM onboard ALOS. Proc. IGARSS 2010, IEEE, CD-ROM.

Takaku, J. and T. Tadono, 2009a. PRISM on-orbit geometric calibration and DSM performance. Transaction on Geoscience and Remote Sensing, IEEE, 47(12), pp. 4060-4073.

Takaku, J. and T. Tadono, 2009b. High resolution DSM generation from ALOS PRISM -Status updates on over three year operations-. Proc. IGARSS 2009, IEEE, CD-ROM.

Takaku, J. and T. Tadono, 2010. High resolution DSM generation from ALOS PRISM -Processing status and influence of attitude fluctuation-. Proc. IGARSS 2010, IEEE, CD-ROM.

Takaku, J. and T. Tadono, 2011. High resolution DSM generation from ALOS PRISM -Archiving and mosaicing-. Proc. IGARSS 2011, IEEE, CD-ROM.

\section{Acknowledgements:}

The authors thank our collaborators from NTT DATA Corp. and RESTEC for participating in the project, in particular, we are grateful to Mr. Junichi Takaku for his technical support and Dr. Ken Tsutsui for his management efforts. 